\title{
Effect of human umbilical cord blood mesenchymal stem cell transplantation on neuronal metabolites in ischemic rabbits
}

\author{
Ye-Ming Guan ${ }^{1}$, Yao Zhu' ${ }^{1}$ Xue-Chun Liu', Hai-Li Huang ${ }^{1}$, Zhi-Wei Wang ${ }^{1}$, Bo Liư ${ }^{2}$, You-Zi Zhu
} and Qing-Song Wang ${ }^{1 *}$

\begin{abstract}
Background: Because there is little research on the effects of transplanted stem cells on neuronal metabolites in infarct areas, we transplanted human umbilical cord blood mesenchymal stem cells (hUCB-MSCs) into cerebral ischemic rabbits and examined the neuronal metabolites.

Results: Rabbits $(n=40)$ were equally divided into sham, middle cerebral artery occlusion (MCAO), hUCB-MSC, and saline groups. The rabbit ischemic model was established by MCAO. The effects of hUCB-MSC transplantation were assessed by proton magnetic resonance spectroscopy ( $\left.{ }^{1} \mathrm{H}-\mathrm{MRS}\right)$, neurological severity scores (NSSs), infarct area volume, neuronal density, and optical density (OD) of microtubule-associated protein 2 (MAP2)-positive cells. We also evaluated complete blood cell counts(CBCs) and serum biochemical parameters. NSSs in the hUCB-MSC group at 7 and 14 days after reperfusion were lower than in MCAO and saline groups $(p<0.05)$. Compared with MCAO and saline groups at 2 weeks after MCAO, the infarction volume in the hUCB-MSC group had decreased remarkably $(p<0.05)$. Significant neuronal metabolic changes occurred in the infarct area at $24 \mathrm{~h}$ and 2 weeks after MCAO. ${ }^{1} \mathrm{H}-\mathrm{MRS}$ revealed an elevation in the lactate (Lac)/creatine including phosphocreatine $(\mathrm{Cr})$ ratio and a decrease in the $\mathrm{N}$-acetylaspartate (NAA)/Cr and choline-containing phospholipids (Cho)/ $\mathrm{Cr}$ ratios at $24 \mathrm{~h}$ after MCAO in the MCAO group $(p<0.01)$. Compared with saline and MCAO groups at $24 \mathrm{~h}$ and 2 weeks after MCAO, NAA/Cr and Cho/Cr ratios had increased significantly, whereas the Lac/Cr ratio had decreased significantly in the hUCB-MSC group $(p<0.01)$. Neuronal density and OD of MAP2-positive cells in the MCAO group were significantly lower than those in the sham group, whereas the neuronal density and OD of MAP2-positive cells in the hUCB-MSC group were higher than those in MCAO and saline groups $(p<0.05)$. CBCs and biochemical parameters were unchanged in the MCAO group at $24 \mathrm{~h}$ and 2 weeks after hUCB-MSC transplantation.
\end{abstract}

Conclusions: Transplanted hUCB-MSCs might ameliorate ischemic damage by influencing neuronal metabolites in the infarct area, providing additional evidence for neuroprotection by stem cells. No significant changes were observed in CBCs or serum biochemical parameters, suggesting that intravenous infusion of hUCB-MSCs is safe for rabbits in the short-term.

Keywords: Human umbilical cord blood mesenchymal stem cells, Cerebral ischemia, Proton magnetic resonance spectroscopy, Middle cerebral artery occlusion

\footnotetext{
* Correspondence: wangqs65@yahoo.com

'Department of Neurology, The 105th Hospital of PLA, Clinic College, Anhui

Medical University, Hefei 230031, China

Full list of author information is available at the end of the article
} 


\section{Background}

Cerebral vascular diseases are a major cause of mortality and disability worldwide. In China, stroke is a leading cause of death and the most common cause of adult disability. At least 1,500,000 patients are affected by cerebrovascular diseases annually, and approximately $30 \%$ of these patients become severely and permanently disabled. Many other survivors are left with permanent functional impairments [1]. Some interventions during the acute phase of stroke such as thrombolytic and neuroprotective agents have been recognized to improve patient outcomes including survival and residual disability. However, once cell damage from stroke has been established, little can be done to restore pre-stroke conditions. In this regard, hundreds of studies have described the therapeutic potential of either endogenous or transplanted stem cells in experimental models of stroke [2]. Therefore, elucidation of potential mechanisms of action is vital to further develop stem cell therapies for translation to the bedside. Various proposed mechanisms have been investigated in preclinical stroke models, including formation of new neuronal circuitry, reductions of apoptosis and inflammation, and promotion of angiogenesis, neurogenesis, and other endogenous repair processes [3-6].

Proton magnetic resonance spectroscopy $\left({ }^{1} \mathrm{H}-\mathrm{MRS}\right)$ is a non-invasive method to detect various neuronal metabolites in real time, including $\mathrm{N}$-acetylaspartate (NAA), creatine including phosphocreatine $(\mathrm{Cr})$, choline-containing phospholipids (Cho), and lactate (Lac) [7]. These advances have led to the emergence of novel approaches to pathophysiological studies of cerebral ischemia [8]. However, little has been reported about how transplanted stem cells might change neuronal metabolites in the infarct area. Thus, we hypothesized that transplanted stem cells may ameliorate ischemic damage by influencing neuronal metabolites in the infarct area. To test this hypothesis, we used a rabbit ischemic model of middle cerebral artery occlusion (MCAO) for transplantation of human umbilical cord blood mesenchymal stem cells (hUCB-MSCs). We then assessed neurological severity scores (NSSs), infarct area volumes, neuronal density, and optical density (OD) of microtubule-associated protein-2 (MAP2)-positive cells. NAA, Cr, Cho, and Lac in the infarct region were measured by ${ }^{1} \mathrm{H}$ - MRS. We also obtained complete blood cell counts (CBCs) and measured serum biochemical parameters.

\section{Results}

\section{Phenotype of hUCB-MSCs}

The cells picked from clones could be passaged successfully and the cells grew as a fusiform or fibroblast-like shape, whereas the subclones developed like a whirlpool (Figure 1).

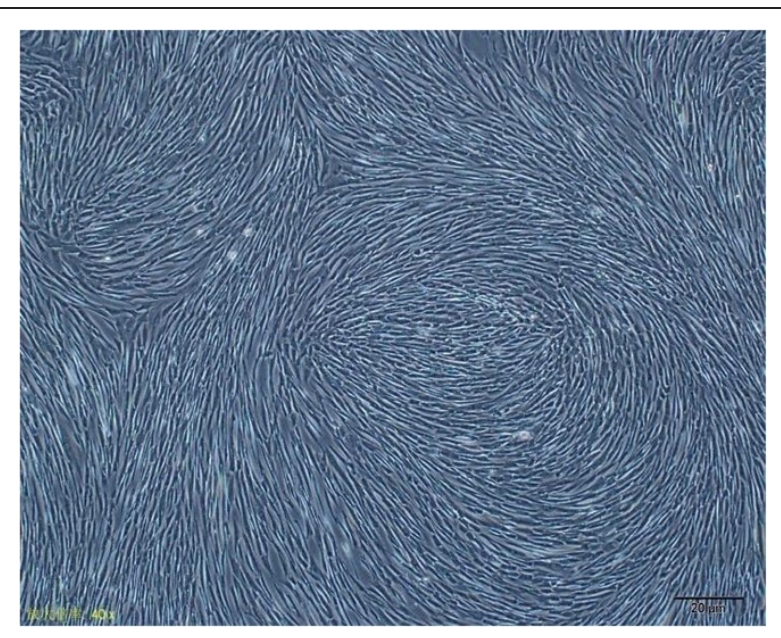

Figure 1 Morphological features of MSCs isolated from hUCB. Passage 5 hUCB-MSCs subcultured for 7 days. The cells exhibited a fusiform or fibroblast-like shape, and were arranged as a whirlpool. Magnification, $\times 40$.

Isolated hUCB-MSCs were characterized by flow cytometric analysis of specific surface antigens. hUCB-MSCs expressed surface antigens CD29 and CD44, but not CD34 and CD45, which was consistent with the surface antigens of bone marrow-derived MSCs (Figure 2).

\section{hUCB-MSC transplantation affects NSSs and the infarct volume in MCAO rabbits}

To evaluate the effect of hUCB-MSCs on neurological deficits after MCAO, we measured NSSs. The NSSs in the hUCB-MSC group at 7 and 14 days after reperfusion were less than those in MACO and saline groups $(p<0.05)$ (Figure 3).

Compared with $\mathrm{MCAO}$ and saline groups at 2 weeks after MCAO, infarct volumes in the hUCB-MSC group decreased remarkably $(p<0.05)$ (Figure 4$)$.

\section{hUCB-MSC transplantation affects neuronal metabolites in MCAO rabbits}

To observe the effects of hUCB-MSC transplantation on neuronal metabolites in the infarct area, ${ }^{1} \mathrm{H}-\mathrm{MRS}$ was used to detect the ratios of NAA/Cr, $\mathrm{Cho} / \mathrm{Cr}$, and $\mathrm{Lac} / \mathrm{Cr}$ at $24 \mathrm{~h}$ and 2 weeks after MCAO. Significant neuronal metabolic changes in the infarct area were observed at $24 \mathrm{~h}$ and 2 weeks after MCAO (Figure 5). ${ }^{1} \mathrm{H}$-MRS spectra showed significant elevation in the $\mathrm{Lac} / \mathrm{Cr}$ ratio and a marked decrease in NAA/Cr and Cho/Cr ratios at $24 \mathrm{~h}$ after MCAO in the MCAO group $(p<0.01$ versus the sham group). Compared with saline and MCAO groups at $24 \mathrm{~h}$ after $\mathrm{MCAO}, \mathrm{NAA} / \mathrm{Cr}$ and $\mathrm{Cho} / \mathrm{Cr}$ ratios were significantly increased and the Lac/Cr ratio was significantly decreased in the hUCB-MSC group $(p<0.01)$ (Figure 6). Interestingly, at 2 weeks after $\mathrm{MCAO}, \mathrm{NAA} / \mathrm{Cr}$ and $\mathrm{Cho} /$ $\mathrm{Cr}$ ratios had increased substantially in the hUCB-MSC 


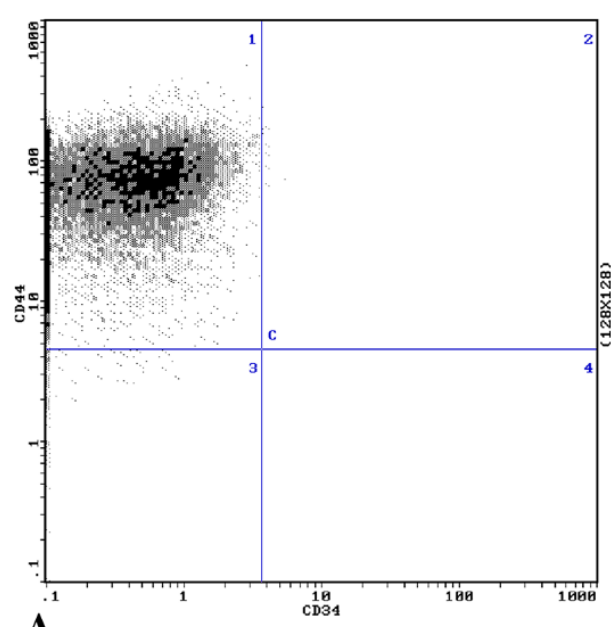

A

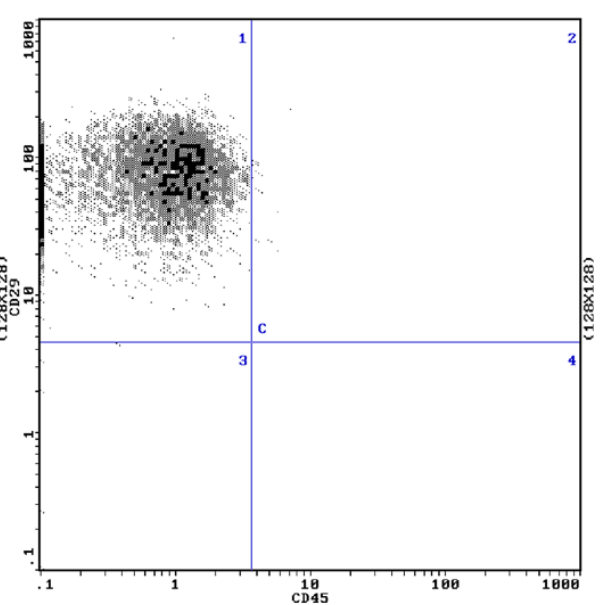

B

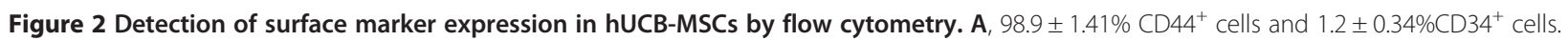
B, $98.7 \pm 1.31 \% \mathrm{CD}_{2} 9^{+}$cells and $1.3 \pm 0.31 \% \mathrm{CD}^{4} 5^{+}$cells.

group, whereas the Lac/Cr ratio has decreased remarkably compared with those in saline and MCAO groups $(p<0.01)$ (Figure 7).

\section{Distribution and migration of hUCB-MSCs in the injured brain}

To investigate whether transplanted hUCB-MSCs could migrate to the infarcted brain area, hUCB-MSCs labeled with 5-bromodeoxyuridine (BrdU) were injected via the right femoral vein of rabbits in the hUCB-MSC group. BrdU-positive cells migrated selectively to the infarction area, which were small with round or oval nuclei containing intensely stained brown chromatin (Figure 8).

\section{hUCB-MSC transplantation affects neuronal density and} OD of MAP2-positive cells in the infarct area

MCAO can cause a complete cerebral infarction as shown by magnetic resonance imaging (MRI) (Figure 9). Cell morphology in the sham group was normal with only a few necrotic cells. More necrotic cells were observed in MCAO and saline groups (Figure 10). MAP2 immunostaining products were localized within the cytodendrites of brain neurons in the sham group. MAP2 immunolabeling was markedly diminished in the infarct zone, and there was an abrupt transition between the infarct and adjacent normal tissue with occasional MAP2-positive cells in the transition zone (Figure 11). To investigate whether transplanted hUCB-MSCs could protect neurons, we examined neuronal density and OD of MAP2-positive cells in the infarct area. Neuronal density and OD of MAP2-positive cells in the MCAO group were significantly lower than those in the sham group $(p<0.05)$. However, neuronal density and OD of MAP2-positive cells in the hUCB-MSC group was higher than those in MCAO and saline groups $(p<0.05)$ (Figures 12 and 13). These data suggest that extensive neuronal damage occurs after MCAO and hUCB-MSC transplantation can prevent ischemic damage.
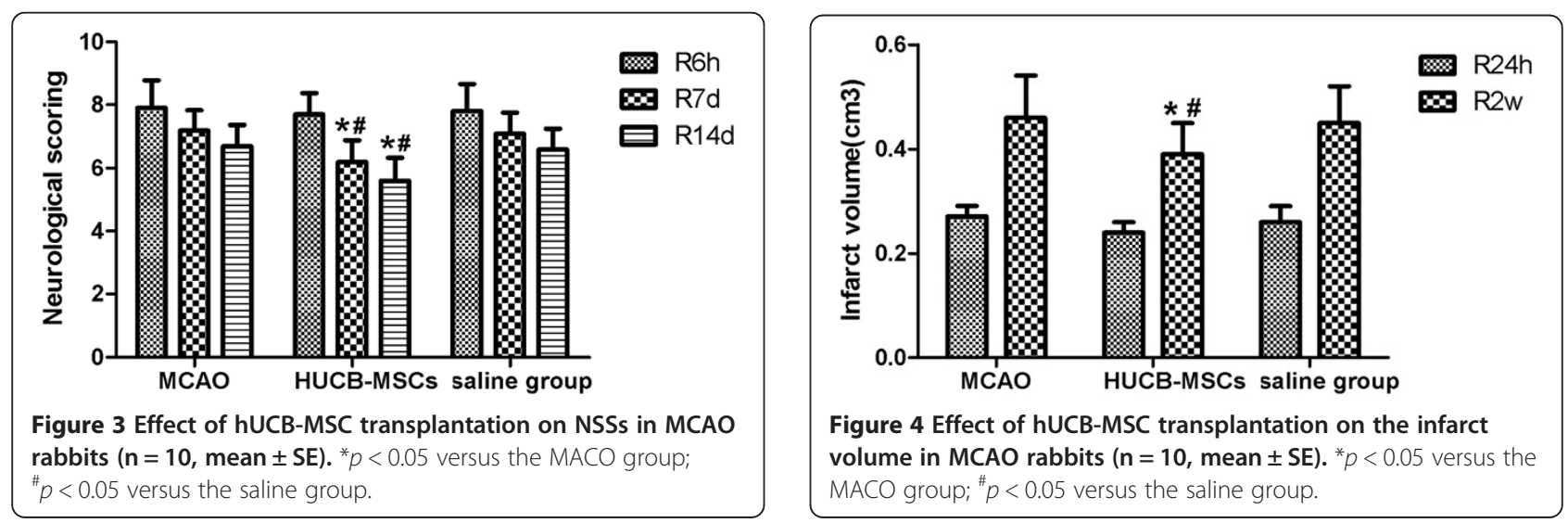

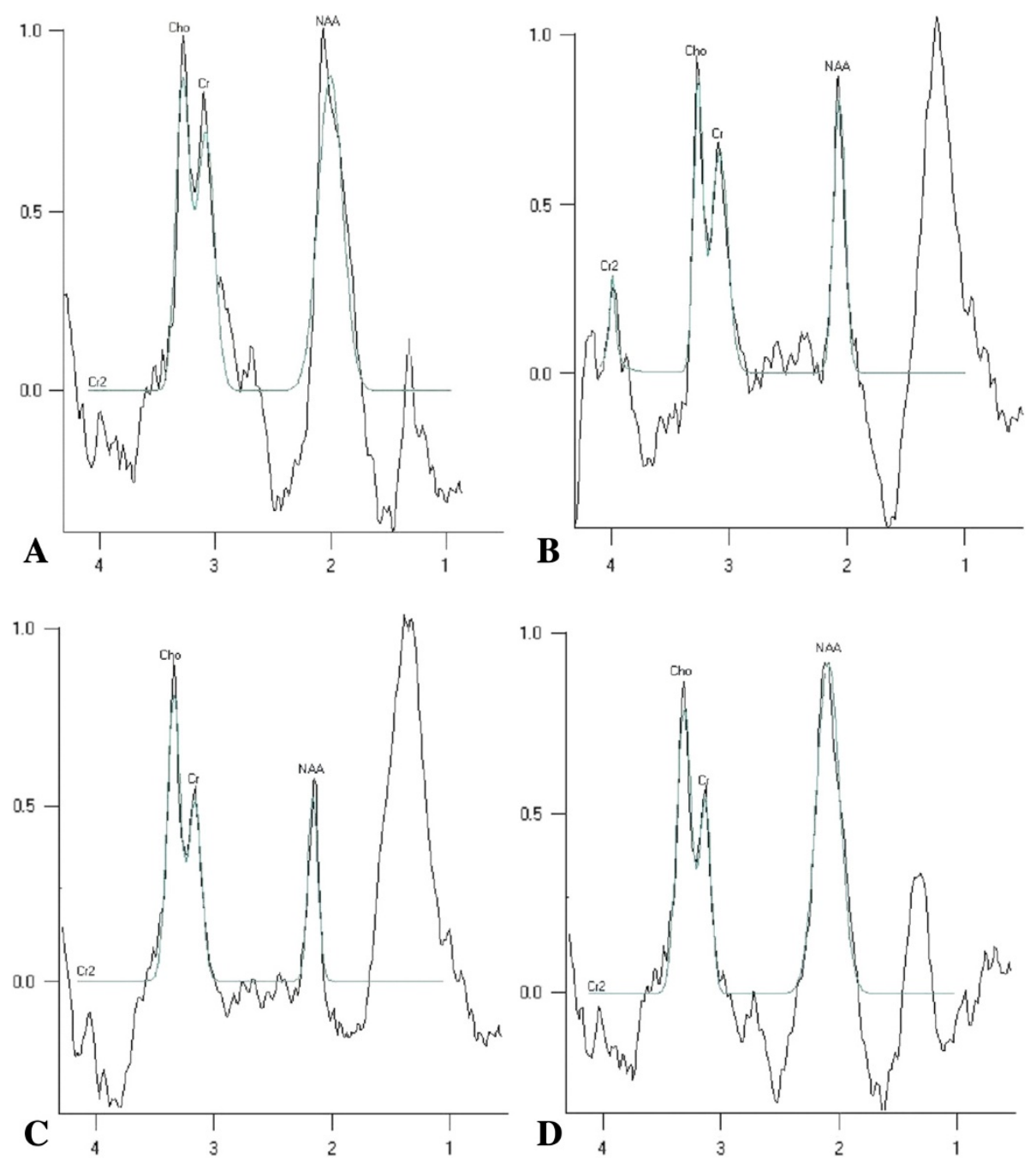

Figure $5{ }^{1} \mathrm{H}$-MRS spectra of the various groups. A) ${ }^{1} \mathrm{H}$-MRS spectra in the sham group had upright steep peaks of NAA and Cho. A Lac peak was not detected. B) ${ }^{1} \mathrm{H}-\mathrm{MRS}$ spectra in the MCAO group revealed a reduction in the NAA peak and distinct upright, steep peaks of Lac were detected at $24 \mathrm{~h}$ after MCAO. C) ${ }^{1} \mathrm{H}$-MRS spectra in the saline group revealed a reduction in the NAA peak. Similar to the saline group, upright steep peaks of Lac were detected at $24 \mathrm{~h}$ after MCAO. D) ${ }^{1} \mathrm{H}-\mathrm{MRS}$ spectra in the hUCB-MSC group at $24 \mathrm{~h}$ after MCAO. Compared with MCAO and saline groups, the NAA peak was higher and the Lac peak was lower.
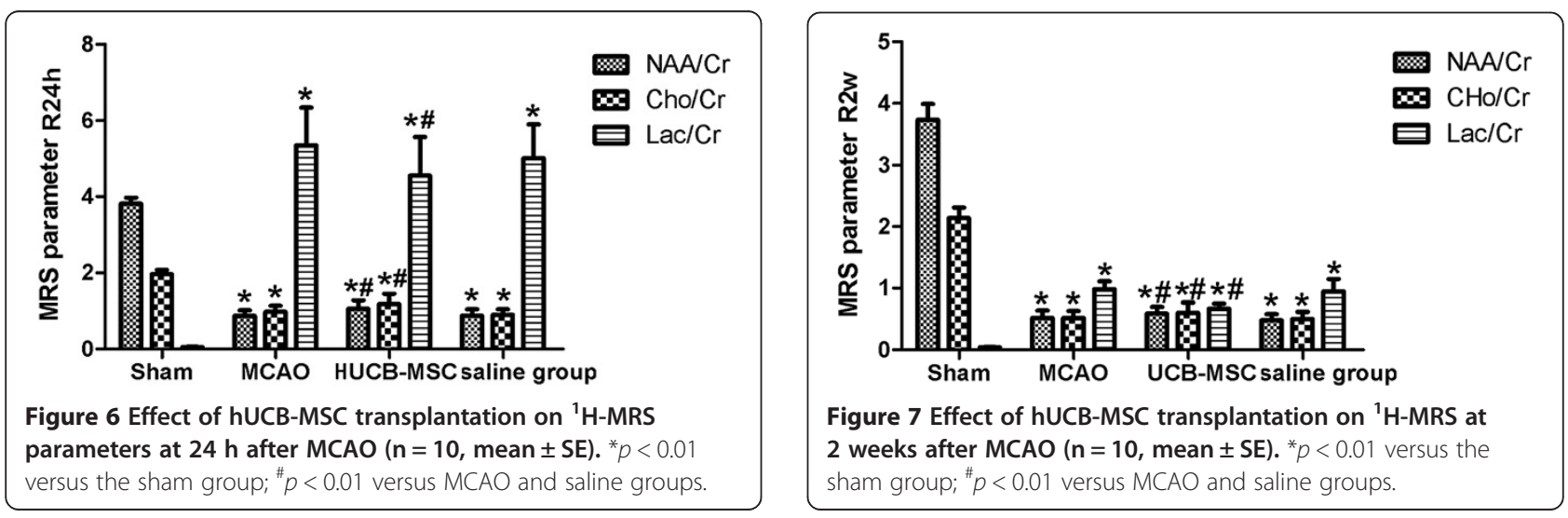


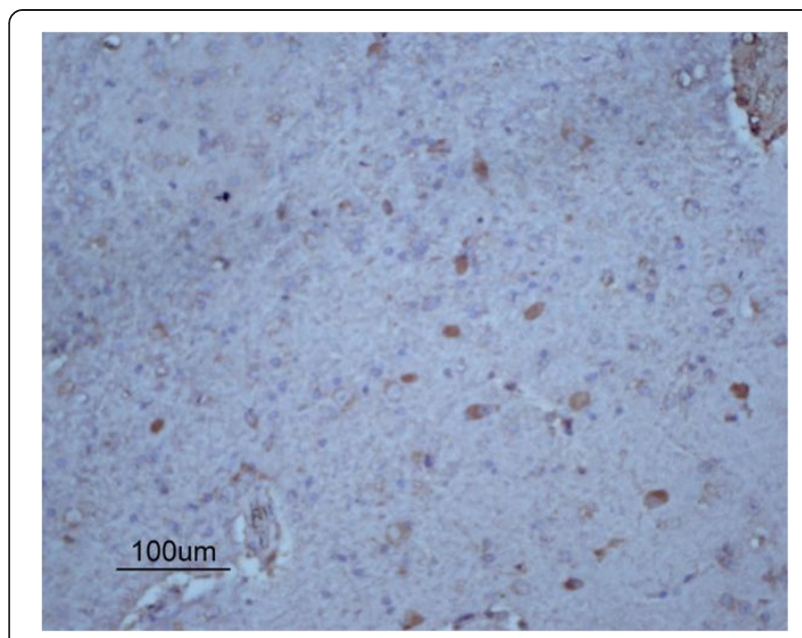

Figure 8 Representative photomicrographs $(\times 100)$ of hUCB-MSCs in the injured brain. BrdU-positive cells were within the ischemic area.

\section{Effects of hUCB-MSC transplantation on CBCs and serum biochemical parameters in MCAO rabbits}

To investigate the safety of hUCB-MSC transplantation in rabbits, $\mathrm{CBCs}$ and serum biochemical parameters were measured at $24 \mathrm{~h}$ and 2 weeks after MCAO. We found no influence on CBCs, liver function, renal function, serum glucose, or serum lipids in MCAO rabbits at $24 \mathrm{~h}$ and 2 weeks after transplantation (Tables 1, 2, 3, 4).

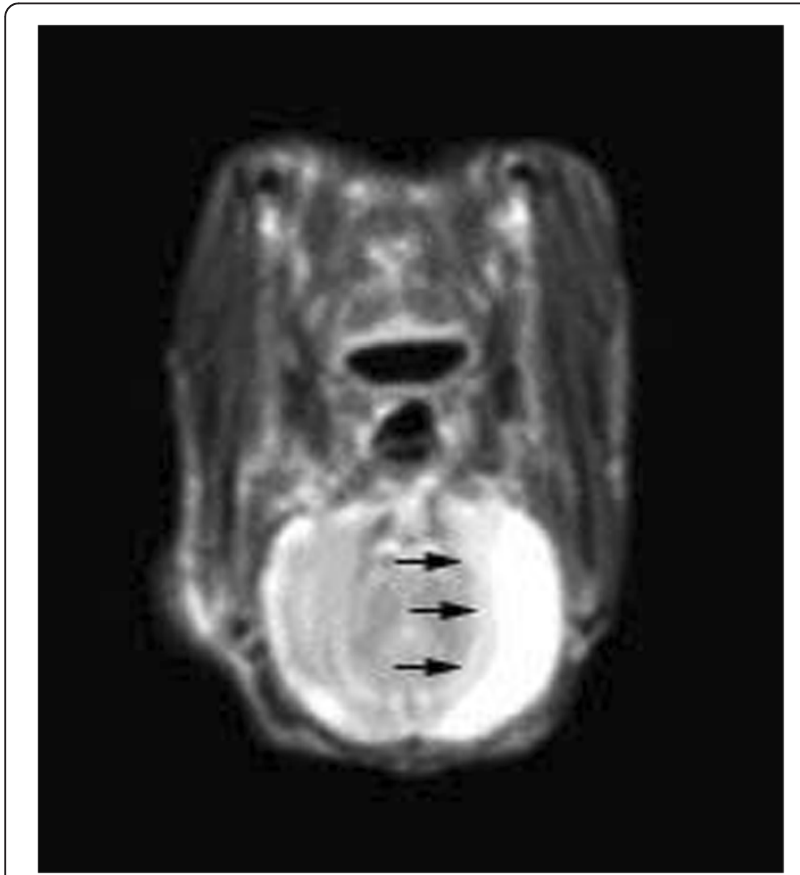

Figure 9 Representative head MRI of MCAO model rabbits. At 2 days after MCAO, T2WI revealed a hyperintensity in the area of the middle cerebral artery. Black arrows indicate the ischemic area.

\section{Discussion}

Stem cell-based strategies are of particular interest in the study of neurological diseases because mature brains have a limited capacity for self-repair. MSCs have great potential to serve as therapeutic agents for stroke treatment, because they are easily obtained and can be expanded rapidly ex vivo for transplantation $[9,10]$. Transplanted MSCs in an ischemic region of the rat brain can differentiate into neural cells and promote functional improvement $[11,12]$. Furthermore, MSCs can improve neurological deficits in stroke patients [13]. hUCB-MSCs have proven to be more advantageous than bone marrow-derived MSCs in terms of cell procurement, storage, and transplantation [14]. Moreover, the number and differentiation ability of bone marrow-derived MSCs significantly decrease with age [15]. Thus, hUCB-MSCs are a better candidate for clinical applications of stem cell-based therapies.

In this study, we confirmed that intravenous infusion of hUCB-MSCs after transient MCAO in rabbits reduced the infarction volume, prevented ischemic neuronal damage, and improved neurological deficits. These results are consistent with previous studies in which beneficial effects were observed in cerebral ischemic models infused with MSCs [16-18].

${ }^{1} \mathrm{H}$-MRS enables serial, non-invasive monitoring of neuronal metabolite disturbances in the diseased brain in vivo, such as Cho, Cr, NAA, and Lac [6]. NAA is located primarily in neuroaxonal tissues. Its presence can be used to determine neuron numbers and activity. Therefore, decreased NAA indicates impaired neuronal and/or axonal function [8]. Cho is a marker of cell reversal and reflects the stability of cell membranes. Its peak level is usually related to cell membrane synthesis and catabolism, which depends on the concentrations of Cho in membrane phospholipids. Cho peaks can reflect the amount of Cho-containing compounds in the myelin membrane, such as phosphocholine and glycerophosphocholine. Abnormal Cho peaks can be observed in pathological conditions such as demyelination, degenerative changes, and ischemic states [19]. Lac is a redox partner of pyruvate and metabolic intermediate between glycolysis and Kreb's or the tricarboxylic acid (TCA) cycle. When oxygen availability is low because of perfusion deficiency or other metabolic stress, the rate of the TCA cycle decreases and pyruvate produced by glycolysis accumulates and is converted to Lac. Thus, Lac can serve as a marker of anaerobic metabolism in stroke. Lac detection by ${ }^{1} \mathrm{H}$-MRS in vivo may allow identification of regions of metabolic stress in the brain and other human tissues, which can potentially identify regional ischemia in stroke [20]. Therefore, serial decreases in $\mathrm{NAA} / \mathrm{Cr}$ and $\mathrm{Cho} / \mathrm{Cr}$ ratios and elevations in the $\mathrm{Lac} / \mathrm{Cr}$ ratio essentially indicate brain damage. In our current study, we observed an elevated Lac/Cr ratio and a marked 


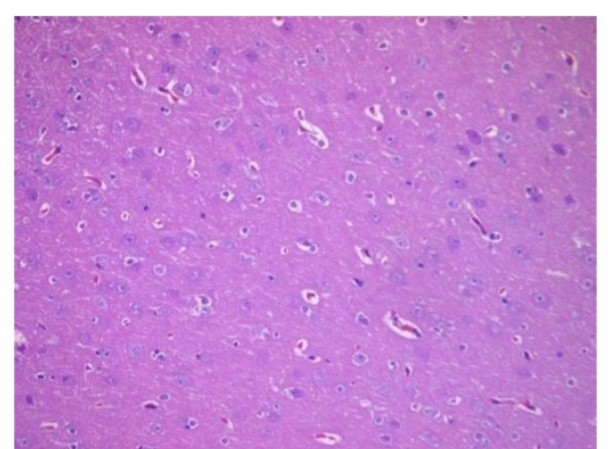

A

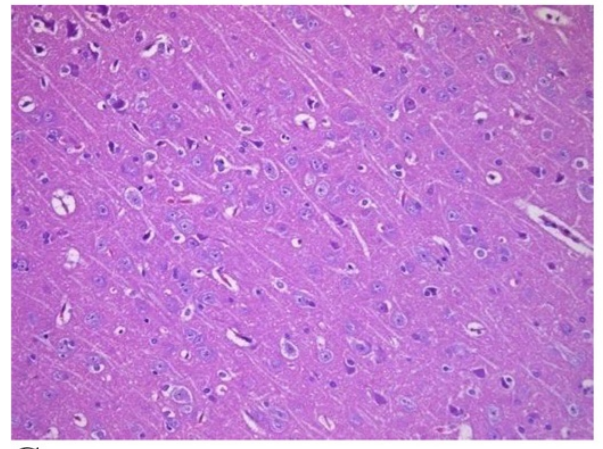

C

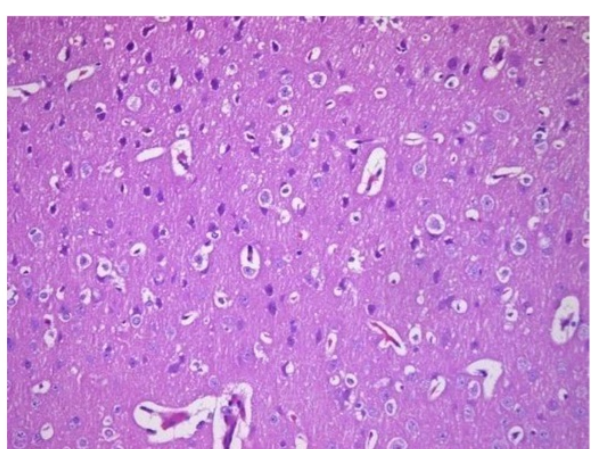

B

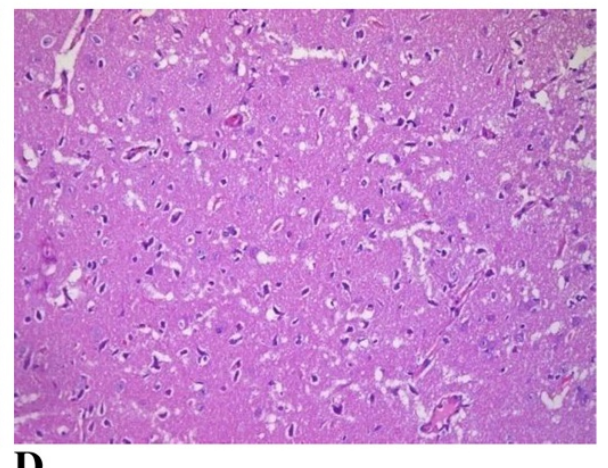

D

Figure 10 Representative photomicrographs (×200) of the infarct area. A, sham group; B, MCAO group; C, hUCB-MSC group; D, saline group.

decrease in $\mathrm{NAA} / \mathrm{Cr}$ and $\mathrm{Cho} / \mathrm{Cr}$ ratios at $24 \mathrm{~h}$ after MCAO in the MCAO group $(p<0.01)$, which was consistent with previous studies [18,21]. However, after intravenous delivery of hUCB-MSCs, ${ }^{1} \mathrm{H}$-MRS analysis at $24 \mathrm{~h}$ and 2 weeks indicated a significant decrease in the $\mathrm{Lac} / \mathrm{Cr}$ ratio and a marked elevation in $\mathrm{NAA} / \mathrm{Cr}$ and $\mathrm{Cho} / \mathrm{Cr}$ ratios compared with those in MCAO and saline groups. These findings suggest neuroprotection induced by the transplantation procedure.

MSCs produce an impressive array of neuroprotective compounds. In a meta-analysis of 60 preclinical studies of intravenously delivered stem cells, Janowski and colleagues reported that good patient outcomes were correlated with inhibition of apoptosis [22]. Neuroprotection by the transplantation procedure may be direct via secretion of neuroprotective compounds or indirect via immunomodulation, angiogenesis, or amplification of the endogenous neural stem cell response [23]. In our present study, hUCB-MSC transplantation restored the ${ }^{1} \mathrm{H}$-MRS data to near normal at 2 weeks after MCAO. Moreover, hUCB-MSC transplantation protected neurons in the infarct area. Thus, our data may offer additional supportive evidence for neuroprotection by stem cells.

The safety of stem cell transplantation for treating ischemia is important. Short-term concerns include acute infusional toxicities to important organs, such as hepatotoxicity and renal toxicity. Long-term concerns include the risk of tumor formation [24]. In our present study, no influence was found on CBCs, liver function, renal function, serum glucose, or serum lipids in MCAO rabbits at $24 \mathrm{~h}$ and 2 weeks after transplantation.

\section{Conclusions}

Taken together, our data show that transplanted hUCBMSCs might ameliorate ischemic damage by influencing neuronal metabolites in the infarct area, which might offer additional supportive evidence for neuroprotection by stem cells. No significant changes were observed in $\mathrm{CBCs}$ or serum biochemical parameters, suggesting that intravenous infusion of hUCB-MSCs is safe for rabbits in the short-term.

\section{Methods}

\section{Rabbit MCAO model}

All animal protocols were approved by the Institutional Animal Care and Use Committee of Clinic College, Anhui Medical University. Male New Zealand white rabbits (provided by the Experimental Animal Center of Anhui Medical University, weighing $2.5-3.5 \mathrm{~kg}$ ) were randomly divided into sham $(n=10)$, MCAO $(n=10)$, hUCB-MSC $(n=10)$, and saline $(n=10)$ groups. Anesthesia was induced with $2 \%$ sodium pentobarbital $(20 \mathrm{mg} / \mathrm{kg})$. The rectal temperature was maintained at $37^{\circ} \mathrm{C}$ throughout the surgical procedure as monitored by an electronic temperature controller linked 


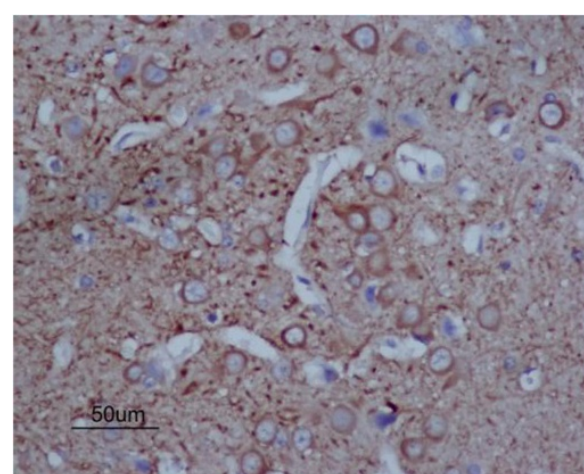

A

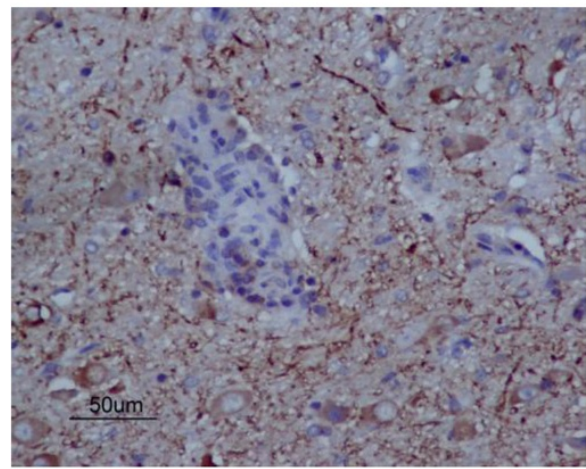

C

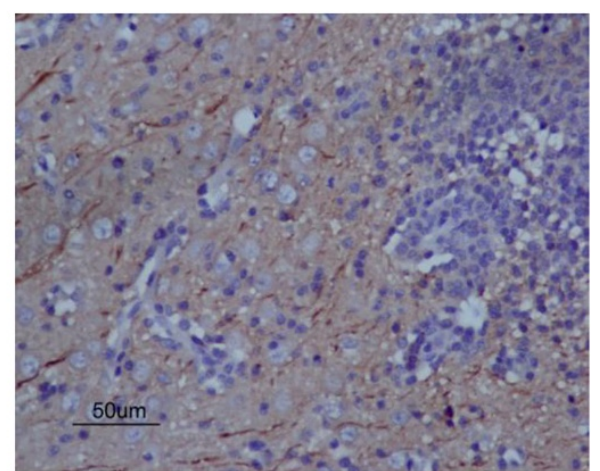

B

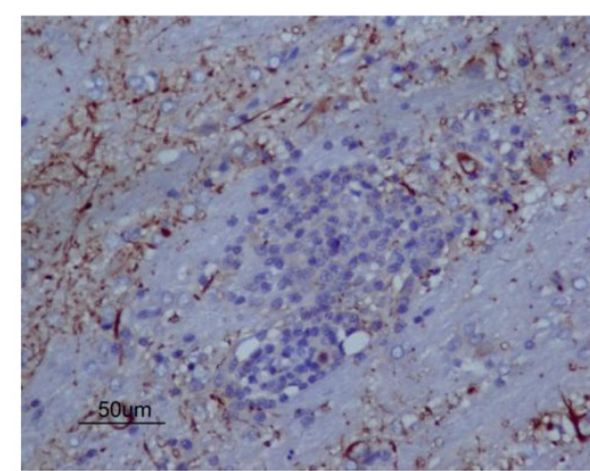

D

Figure 11 Representative photomicrographs (×200) of MAP2 immunohistochemistry. A, sham group; B, MCAO group; C, hUCB-MSC group; D, saline group.

to a heating pad (FHC, Bowdoinham, ME, USA). Transient MCAO was induced as described previously [25]. Briefly, the right common carotid artery (CCA), external carotid artery, and internal carotid artery (ICA) were exposed through a ventral midline incision. A nylon monofilament suture with a rounded tip (head-end diameter: $0.53 \mathrm{~mm}$ ) was introduced into the CCA lumen and gently advanced into the ICA until it blocked the bifurcating origin of the MCA. At $2 \mathrm{~h}$ after occlusion, the rabbits were

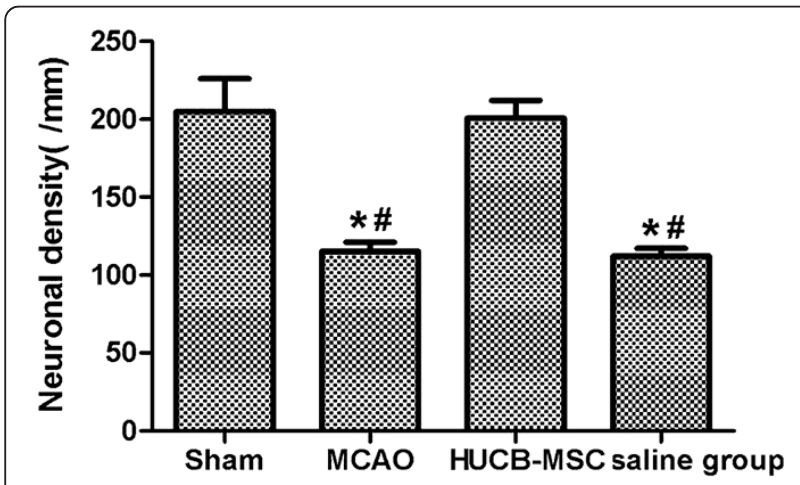

Figure 12 Neuronal density (per $\left.\mathrm{mm}^{2}\right)$ among groups $(n=10$, mean \pm SE). ${ }^{*} p<0.05$ versus the sham group; ${ }^{\#} p<0.05$ versus $M C A O$ and saline groups. re-anesthetized and reperfused by withdrawing the suture until its tip cleared the lumen of the CCA.

\section{hUCB-MSC culture and identification}

Ethical approval for the use of hUCB-MSCs was obtained from Clinic College, Anhui Medical University.

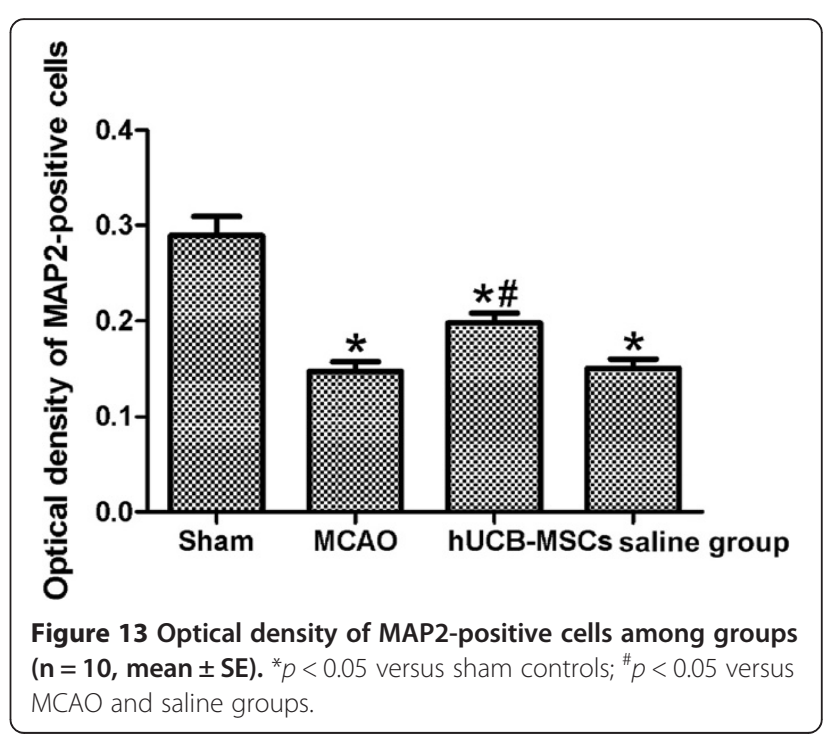


Table 1 CBCs of each group at $24 \mathrm{~h}$ after MCAO $(n=10$, mean $\pm \mathrm{SE}$ )

\begin{tabular}{|c|c|c|c|c|}
\hline & $\begin{array}{l}\text { WBCs } \\
\left(10^{9} / \mathrm{L}\right)\end{array}$ & $\begin{array}{c}\text { RBCs } \\
\left(10^{12} / \mathrm{L}\right)\end{array}$ & HB (g/L) & PLT $\left(10^{9} / L\right)$ \\
\hline Sham & $7.56 \pm 1.40$ & $5.05 \pm 0.55$ & $120.50 \pm 17.04$ & $241.90 \pm 56.58$ \\
\hline MCAO & $7.74 \pm 1.16$ & $5.36 \pm 0.73$ & $115.00 \pm 14.94$ & $258.00 \pm 68.81$ \\
\hline hUCB-MSC & $7.50 \pm 1.30$ & $5.22 \pm 0.70$ & $112.10 \pm 13.07$ & $281.30 \pm 88.51$ \\
\hline Saline & $7.50 \pm 1.92$ & $5.33 \pm 0.62$ & $119.60 \pm 20.80$ & $240.50 \pm 63.31$ \\
\hline
\end{tabular}

Note: white blood cells (WBCs); red blood cells (RBCs); hemoglobin (HB); platelet count (PLT).

Human UCB samples $(60-80 \mathrm{ml})$ were collected from the umbilical vein of full-term infants during deliveries with informed maternal consent. Isolation and expansion of hUCB-MSCs was conducted as reported previously [26]. Briefly, mononuclear cells were isolated by density gradient centrifugation. The separated mononuclear cells were washed, resuspended in Dulbecco's modified Eagle's medium, and then seeded at $1 \times 10^{6}$ cells $/ \mathrm{mL}$. Cultures were maintained at $37^{\circ} \mathrm{C}$ in a humidified atmosphere containing 5\% $\mathrm{CO}_{2}$, and the culture medium was changed twice a week. Fibroblast-like adherent cells were observed in UCB-derived mononuclear cell cultures. After $1-3$ weeks, MSC colonies reached $80 \%$ confluence and the cells were trypsinized ( $0.25 \%$ trypsin), washed, resuspended in culture medium, and subcultured at $5 \times 10^{4}$ cells $/ \mathrm{cm}^{2}$. MSCs of each hUCB harvest were expanded ex vivo by successive subcultivation under the same conditions. Passage 5-8 cells with a more than 1,000-fold expanding capacity were used in experiments.

Isolated hUCB-MSCs were characterized by flow cytometric analysis of specific surface antigens using CD29FITC, CD45-PE, CD44-FITC, and CD34-PE monoclonal antibodies (BD Bioscience, San Jose, CA, USA). The cells were incubated in the dark at room temperature for $20 \mathrm{~min}$.

Table 2 Serum biochemistry of each group at $24 \mathrm{~h}$ after MCAO ( $n=10$, mean \pm SE)

\begin{tabular}{lcccc}
\hline & Sham & MCAO & hUCB-MSC & Saline \\
\hline ALT (U/L) & $88.30 \pm 24.74$ & $85.20 \pm 22.95$ & $95.40 \pm 22.20$ & $88.80 \pm 17.93$ \\
AST (U/L) & $90.50 \pm 6.85$ & $95.20 \pm 18.82$ & $95.40 \pm 18.39$ & $90.00 \pm 15.89$ \\
Urea & $5.93 \pm 1.03$ & $6.06 \pm 0.95$ & $5.68 \pm 1.26$ & $5.65 \pm 1.03$ \\
$(\mathrm{mmol} / \mathrm{L})$ & & & & \\
$\mathrm{Cr}(\mu \mathrm{mol} / \mathrm{L})$ & $90.20 \pm 24.89$ & $86.50 \pm 12.87$ & $92.10 \pm 14.43$ & $94.70 \pm 11.69$ \\
$\mathrm{TG}$ & $0.84 \pm 0.24$ & $1.11 \pm 0.81$ & $1.18 \pm 0.83$ & $1.26 \pm 1.12$ \\
$(\mathrm{mmol} / \mathrm{L})$ & & & & \\
Chol & $1.89 \pm 0.75$ & $1.57 \pm 0.83$ & $1.50 \pm 0.74$ & $1.74 \pm 0.57$ \\
$(\mathrm{mmol} / \mathrm{L})$ & & & & \\
Glu (mmol/L) & $8.07 \pm 0.91$ & $7.98 \pm 0.85$ & $8.44 \pm 1.35$ & $8.41 \pm 1.47$
\end{tabular}

Note: aspartatenine aminotransferase (ALT); aspartate aminotransferase (AST).
Table 3 CBCs of each group at 2 weeks after MCAO $(n=10$, mean $\pm S E)$

\begin{tabular}{lcccc}
\hline & $\begin{array}{c}\text { WBCs } \\
\left(\mathbf{1 0 ^ { 9 }} / \mathbf{L}\right)\end{array}$ & $\begin{array}{c}\text { RBCs } \\
\left(\mathbf{1 0 ^ { 1 2 }} / \mathbf{L}\right)\end{array}$ & HB (g/L) & PLT (109/L) \\
\hline Sham & $7.32 \pm 1.28$ & $5.21 \pm 0.46$ & $122.52 \pm 14.07$ & $245.60 \pm 53.21$ \\
MCAO & $7.57 \pm 1.41$ & $5.62 \pm 0.23$ & $114.40 \pm 13.26$ & $255.00 \pm 70.07$ \\
hUCB-MSC & $7.28 \pm 1.08$ & $5.34 \pm 0.41$ & $112.06 \pm 13.64$ & $277.30 \pm 85.65$ \\
Saline & $7.33 \pm 1.15$ & $5.77 \pm 0.52$ & $117.63 \pm 18.83$ & $270.50 \pm 82.77$
\end{tabular}

Note: white blood cells (WBCs); red blood cells (RBCs); hemoglobin (HB); platelet count (PLT).

Labeling of hUCB-MSCs with BrdU and tracing BrdUpositive cells in the injured brain

hUCB-MSCs at passage 5 were cultured in complete growth medium under the same conditions described above. Cells were labeled by incubation in medium containing $5 \mathrm{mmol} / \mathrm{L} \mathrm{BrdU}$ (Roche Diagnostics, Mannheim, Germany) for $48 \mathrm{~h}$ before harvest. BrdU was added according to the manufacturer's instructions. BrdU binds to DNA and labels the nucleus, serving as a tracer for in vivo studies of transplanted cells. The distribution of BrdU-positive cells was detected by immunohistochemical methods [27]. After 2 weeks, serial sections (6 $\mu \mathrm{m}$ thick) through the ischemic cortex were prepared from each rabbit. The sections were then incubated with a primary anti-BrdU monoclonal antibody (1:500) and then pero xidase-conjugated anti-rabbit IgG as the secondary antibody $(1: 100)$. The cells were stained with diaminobenzidine as the chromagen.

\section{hUCB-MSC transplantation}

Passage 5 hUCB-MSCs were injected intravenously within several minutes after MCAO. hUCB-MSCs $\left(5 \times 10^{6}\right)$ diluted in $2 \mathrm{~mL}$ PBS were injected slowly for $5 \mathrm{~min}$ via the right leg femoral vein. For the saline group, $2 \mathrm{~mL}$ PBS without hUCB-MSCs was injected in the same manner.

Table 4 Serum biochemistry of each group at 2 weeks after MCAO ( $n=10$, mean $\pm S E)$

\begin{tabular}{lcccc}
\hline & Sham & MACO & hUCB-MSC & Saline \\
\hline ALT (U/L) & $78.40 \pm 21.12$ & $82.50 \pm 24.05$ & $88.80 \pm 23.36$ & $90.50 \pm 19.34$ \\
AST(U/L) & $89.60 \pm 16.75$ & $91.70 \pm 18.52$ & $92.40 \pm 17.45$ & $92.20 \pm 15.79$ \\
Urea & $5.71 \pm 1.05$ & $5.56 \pm 0.87$ & $5.78 \pm 1.03$ & $5.47 \pm 0.76$ \\
(mmol/L) & & & & \\
Cr ( $\mathrm{mmol} / \mathrm{L})$ & $87.20 \pm 15.43$ & $89.86 \pm 15.34$ & $90.70 \pm 14.23$ & $92.40 \pm 13.64$ \\
TG (mmol/L) & $0.75 \pm 0.17$ & $1.25 \pm 0.61$ & $1.27 \pm 0.47$ & $1.04 \pm 0.26$ \\
Chol & $1.76 \pm 0.86$ & $1.79 \pm 0.45$ & $1.67 \pm 0.38$ & $1.58 \pm 0.62$ \\
(mmol/L) & & & & \\
Glu (mmol/L) & $7.47 . \pm 0.91$ & $8.03 \pm 0.25$ & $8.23 \pm 1.27$ & $7.61 \pm 0.83$ \\
\hline
\end{tabular}

Note: aspartatenine aminotransferase (ALT); aspartate aminotransferase (AST). 


\section{Neurological deficit scale}

Neurological deficits were measured in an independent and blinded fashion by NSSs as described by Purdy [28]. NSSs include motor function, consciousness, head turning, circling, and hemianopsia. The minimum NSS is 2, which is normal, and the maximal NSS is 11, indicating a comatose or dead animal. NSSs were measured at $6 \mathrm{~h}$, 7 days, and 14 days after hUCB-MSC transplantation.

\section{${ }^{1} \mathrm{H}-\mathrm{MRS}$ examination}

${ }^{1} \mathrm{H}$-MRS data were obtained using a 1.5 Tesla MR machine (Siemens 1.5 T Novus MR scanner) and the point resolved echo spectroscopy method for localization and $5 \mathrm{~mm} \times 5 \mathrm{~mm} \times 5 \mathrm{~mm}$ volume of interest of the hippocampus in the bilateral temporal lobe. TE, TR, and NEX were 18, 400, and $200 \mathrm{~ms}$, respectively. The ${ }^{1} \mathrm{H}$-MRS detection technology used was multi-voxel spectroscopy sequence of fixed-point resolution data acquisition. All spectral data were automatically processed in probe sequence on the MR console. Probe acquisition protocols employed in this study included ratio frequency transmitter receiver gain adjustment, water suppression, auto-shim process, and data acquisition. The area of the peak for each organic compound and its comparative value were obtained for each volume of interest. Four major peaks were measured individually and determined to be Cho (3.2 ppm), Cr (3.0 ppm), NAA (2.0 ppm), and Lac (1.3 ppm), which were measured individually. Peak areas were expressed as the relative ratio to $\mathrm{Cr}$ in each spectrum. Cr was used as the reference because of its relative stability, even under rapid energy metabolism fluctuations [29]. Infarct volumes were automatically calculated with Tools Free-hand ROI software (Siemens).Two neuroradiologists analyzed all ${ }^{1} \mathrm{H}-\mathrm{MRS}$ and MRI data.

\section{Histopathological studies}

At 2 weeks after MCAO, all rabbits were anesthetized with $20 \%$ urethane and then their brains were quickly removed and fixed with $10 \%$ formalin. After $24 \mathrm{~h}$, the tissues were embedded in paraffin and serial sections (6 $\mu \mathrm{m}$ thick) were prepared through the ischemic cortex. The sections were then stained with hematoxylin and eosin. Neurons in a linear length $(1 \mathrm{~mm})$ were counted under a light microscope.

\section{MAP2 immunohistochemistry}

The sections were dewaxed with a standard procedure and washed with PBS as described previously [30]. Briefly, after blocking in 10\% normal goat serum/PBS for $30 \mathrm{~min}$ at room temperature, the sections were incubated with a mouse anti-MAP-2 polyclonal antibody (1:200, Santa Cruz Biotechnology, Santa Cruz, CA, USA) for $16 \mathrm{~h}$ at $4^{\circ} \mathrm{C}$. After three washes in PBS (10 min each), the sections were incubated with a goat anti-mouse secondary antibody for
$2 \mathrm{~h}$ at room temperature. The sections were washed as described above and then incubated with an avidin biotinylated enzyme complex for $1 \mathrm{~h}$ at room temperature. The sections were visualized with diaminobenzidine. Nuclei were counterstained with hematoxylin. Finally, the sections were dehydrated in an alcohol gradient and cleared with xylene. Ten images were then randomly captured in microscopic fields for each group. The images were used to analyze the OD of MAP2-positive cells with JEDA 801D software (Jiangsu JEDA Science Technology Development, China).

\section{Statistical analysis}

All data were expressed as the means \pm standard error (SE). The Student's t-test was used to compare differences between two groups. One-way analysis of variance was used to determine the significance of differences among multiple comparisons. Statistical significance was set at $p<0.05$.

\section{Competing interests}

The authors declare that they have no competing interests.

\section{Authors' contributions}

YM, YZ, ZW, and XC established the ischemia model, helped with sample preparation, measured NSSs, and performed histopathological studies and immunohistochemistry. BL performed cell cultures. $Y Z$ and $H L$ measured the infarct area volume and performed ${ }^{1} \mathrm{H}-\mathrm{MRS}$ measurements. $Y M, Y Z, H L$, and QS drafted the manuscript. QS, and YZ conceptualized the study and participated in its design and coordination. All authors read and approved the final manuscript.

\section{Acknowledgements}

This work was supported by a grant from the Natural Science Foundation of China (81171108).

\section{Author details}

'Department of Neurology, The 105th Hospital of PLA, Clinic College, Anhui Medical University, Hefei 230031, China. ${ }^{2}$ Center for Cell Therapy, The 105th Hospital of PLA, Clinic College, Anhui Medical University, Hefei 230031, China. ${ }^{3}$ Department of Radiology, The 105th Hospital of PLA, Clinic College, Anhui Medical University, Hefei 230031, China.

Received: 15 July 2013 Accepted: 12 March 2014

Published: 17 March 2014

\section{References}

1. Yang G, Wang Y, Zeng Y, Gao GF, Liang X, Zhou M, Wan X, Yu S, Jiang Y, Naghavi M, Vos T, Wang H, Lopez AD, Murry CJ: Rapid health transition in China, 1990-2010: findings from the global burden of disease study 2010. Lancet 2013, 381:1987-2015.

2. Burns TC, Verfaillie CM, Low WC: Stem cells for ischemic brain injury: a critical review. J Comp Neurol 2009, 515:125-144.

3. Banerjee S, Williamson DA, Habib N, Chataway J: The potential benefit of stem cell therapy after stroke: an update. Vasc Health Risk Manag 2012, 8:569-580.

4. Lim JY, Jeong CH, Jun JA, Kim SM, Ryu CH, Hou Y, Oh W, Chang JW, Jeun SS: Therapeutic effects of human umbilical cord blood-derived mesenchymal stem cells after intrathecal administration by lumbar puncture in a rat model of cerebral ischemia. Stem Cell Res Ther 2011, 2:38-51.

5. Chen J, Li Y, Wang L, Lu M, Zhang X, Chopp M: Therapeutic benefit of intravenous administration of bone marrow stromal cells after cerebral ischemia in rats. Stroke 2001, 32:1005-1011.

6. Bacigaluppi M, Pluchino S, Jametti LP, Kilic E, Kilic U, Salani G, Brambilla E, West MJ, Comi G, Martino G, Hermann DM: Delayed post-ischaemic 
neuroprotection followingsystemic neural stem cell transplantation involvesmultiple mechanisms. Brain 2009, 132:2239-2251.

7. Harada K, Honmoua O, Liua H, Bandob M, Houkina K, Kocsisc JD: Magnetic resonance lactate and lipid signals in rat brain after middle cerebral artery occlusion model. Brain Res 2007, 1134:206-213.

8. Kenshi T, Hironaka I, Masaki H, Hitoshi M, Masatoyo N, Tsutomu N: Serial assessments of delayed encephalopathy after carbon monoxide poisoning using magnetic resonance spectroscopy and diffusion tensor imaging 3.0 T system. Eur Neurol 2008, 59:55-61.

9. Caplan Al: Mesenchymal stem cells. J Orthop Res 1991, 9:641-650.

10. Lee J, Kuroda S, Shichinohe H, Ikeda J, Seki T, Hida K, Tada M, Sawada K, Iwasaki Y: Migration and differentiation of nuclear fluorescence-labeled bone marrow stromal cells after transplantation into cerebral infarct and spinal cord injury in mice. Neuropathology 2003, 23:169-180.

11. Keating A: Mesenchymal stromal cells. Curr Opin Hematol 2006, 13:419-425.

12. Shichinohe H, Kuroda S, Yano S, Ohnishi T, Tamagami H, Hida K, Iwasaki Y: Improved expression of gamma-aminobutyric acid receptor in mice with cerebral infarct and transplanted bone marrow stromal cells: an autoradiographic and histologic analysis. J Nucl Med 2006, 47:486-491.

13. Bang OY, Lee JS, Lee PH, LEE G: Autologous mesenchymal stem cell transplantation in stroke patients. Ann Neurol 2005, 57:874-882.

14. Lindvall O, Kokaia Z, Martinez-Serrano A: Stem cell therapy for human neurodegenerative disorders-how to make it work. Nat Med 2004, 10:42-50.

15. Jeong JA, Hong SH, Gang EJ, Ahn C, Hwang SH, Yang IH, Han H, Kim H: Differential gene expression profiling of human umbilical cord blood derived mesenchymal stem cells by DNA microarray. Stem Cells 2005, 23:584-593.

16. lihoshi S, Honmou O, Houkin K, Hashi K, Kocsis JD: A therapeutic window for intravenous administration of autologous bone marrow after cerebral ischemia in adult rats. Brain Res 2004, 1007:1-9.

17. Kurozumi K, Nakamura K, Tamiya T, Kawano Y, Kobune M, Hirai S, Uchida H, Sasaki K, Ito Y, Kato K, Honmou O, Houkin K, Date I, Hamada H: BDNF gene-modified mesenchymal stem cells promote functional recovery and reduce infarct size in the rat middle cerebral artery occlusion model. Mol Ther 2004, 9:189-197.

18. Li Y, Chen J, Chen XG, Wang L, Gautam SC, Xu YX, Katakowski M, Zhang L, Lu M, Janakiraman N, Chopp M: Human marrow stromal cell therapy for stroke in rat: neurotrophins and functional recovery. Neurology 2002, 59:514-523.

19. Wang Q, Guan Y, Liu X, Liu H, Wang J: The free radical scavenger, edaravone, ameliorates delayed neuropsychological sequelae after acute carbon monoxide poisoning in rabbits. Undersea Hyperbaric Med 2013, 40:219-225.

20. Abe K, Yoshimura H, Tanaka H, Fujita N, Hikita T, Sakoda S: Comparison of conventional and diffusion weighted MRI and proton MR spectroscopy in patients with mitochondrial encephalomyopathy, lactic acidosis, and stroke-like events. Neuroradiology 2004, 46:113-117.

21. Honmaa T, Honmou O, lihoshia S, Haradaa K, Houkina K, Hamadab H, Kocsis JD: Intravenous infusion of immortalized human mesenchymal stem cells protects against injury in a cerebral ischemia model in adult rat. Exp Neurol 2006, 199:56-66.

22. Janowski M, Walczak P, Date I: Intravenous route of cell delivery for treatment of neurological disorders: a meta-analysis of preclinical results. Stem Cells Dev 2010, 19:5-16.

23. Burns TC, Steinberg GK: Stem cells and stroke: opportunities, challenges, and strategies. Expert Opin Biol Ther 2011, 11:447-461.

24. Stem Cell Therapies as an Emerging Paradigm in Stroke participants: Stem Cell Therapies as an Emerging Paradigm in Stroke (STEPS): bridging basic and clinical science for cellular and neurogenic factor therapy in treating stroke. Stroke 2009, 40:510-515.

25. Aronowski J, Strong R, Grotta JC: Reperfusion injury: demonstration of brain damage produced by reperfusion after transient focal ischemia in rats. J Cereb Blood Flow Metab 1997, 17:1048-1056.

26. Yang $S E, H a C W$, Jung $M$, Jin HJ, Lee M, Song H, Choi S, Oh W, Yang YS: Mesenchymal stem/progenitor cells developed in cultures from UC blood. Cytotherapy 2004, 6:476-486.

27. Luo G, Cheng W, He W, Wang X, Tan J, Fitzgerald M, Li X, Wu J: Promotion of cutaneous wound healing by local application of mesenchymal stem cells derived from human umbilical cord blood. Wound Rep Reg 2010, 18:506-513.
28. Purdy PD, Devous MD, Batjer HH, White CL, Meyer Y, Samson DS: Microfibrillar collagen model of canine cerebral infarction. Stroke 1989, 20:1361-1367.

29. Kondziella D, Danielsen ER, Hansen K, Thomsen C, Jansen EC, Arlien-Soeborg P: $\mathrm{H}^{1}-\mathrm{MR}$ spectroscopy of gray and white matter in carbon monoxide poisoning. J Neuro/ 2009, 256:970-979.

30. Feng HL, Yan L, Cui LY: Effects of repetitive transcranial magnetic stimulation on adenosine triphosphate content and microtubule associated protein-2 expression after cerebral ischemia-reperfusion injury in rat brain. Chin Med J 2008, 121:1307-1312.

doi:10.1186/1471-2202-15-41

Cite this article as: Guan et al:: Effect of human umbilical cord blood mesenchymal stem cell transplantation on neuronal metabolites in ischemic rabbits. BMC Neuroscience 2014 15:41.

\section{Submit your next manuscript to BioMed Central and take full advantage of:}

- Convenient online submission

- Thorough peer review

- No space constraints or color figure charges

- Immediate publication on acceptance

- Inclusion in PubMed, CAS, Scopus and Google Scholar

- Research which is freely available for redistribution 